\title{
Numerical Analysis of Maneuvering Rotorcraft Using Moving Overlapped Grid Method
}

\author{
By Choongmo YANG and Takashi AOYAMA \\ Japan Aerospace Exploration Agency (JAXA), Chofu, Japan
}

(Received July 23rd, 2007)

\begin{abstract}
In transient flight, rotor wakes and tip vortex generated by unsteady blade air-loads and blade motions are fully unsteady and 3-dimensionally-aperiodic, giving rise to significant complicity in accurate analysis compared to steady flight. We propose a hybrid approach by splitting the motions of a maneuvering helicopter into translation and rotation. Translation is simulated using a non-inertial moving (translating) coordinate for which new governing equations are derived, and rotations are simulated by moving each grid in the frame. A flow simulation (CFD) code is constructed by using the hybrid approach, then two simple cases (accelerating/decelerating flight and right-turn flight) for maneuvering helicopter are calculated using the moving overlapped grid method, which is now one of the most advanced techniques for tip-vortex capture. The vortex bundling phenomena, which is a main characteristic of right-turn flight, is well captured by the simulation code. The results of the present study provide better understanding of the characteristics for maneuvering rotorcraft, which can be valuable in full helicopter design.
\end{abstract}

Key Words: $\quad$ Rotor Wake, Maneuver, CFD, Overlapped Grid System

\section{Nomenclature}

$\vec{V}:$ absolute velocity in inertial frame $X Y Z$

$\vec{V}_{\mathrm{r}}$ : relative velocity in non-inertial frame $x y z$

$\vec{V}_{\mathrm{t}}$ : translation velocity of origin of $x y z$

$\vec{V}_{\mathrm{T}}$ : transformation velocity of $x y z$ from absolute to relative motion

$\vec{\varpi}$ : angular velocity of rotating non-inertial frame $x y z$

$e$ : energy for fixed (inertial) reference coordinate

$h$ : enthalpy for fixed (inertial) reference coordinate

$E$ : roenergy for constant-moving reference coordinate

$H$ : rothalpy for constant-moving reference coordinate

$R$ : radius of rotor blade

$d$ : distance from the center of rotor rotation

\section{Introduction}

Runway-independent aircraft, such as helicopters and tiltrotor aircrafts, are used in various fields, such as emergency medical service (EMS), fast and efficient passenger and cargo shuttles, fire fighting, disaster relief, surveillance missions, because of its aviation capabilities in hovering and VTOL. However, noise, cost, and visual flight rules (VFR) problems prevent helicopters from being widely used as inter-city transportation in densely populated areas. Many researchers are researching much wider use of helicopters in the future. The FAA is promoting the Rotorcraft Research and Development Initiative 10-year Plan ${ }^{1)}$ with the objective of developing and demonstrating technologies to enable highly reliable, cost-effective, and environmentally friendly rotorcraft. Also Europe is running the FriendCOPTER Project ${ }^{2)}$ as an integrated research project aiming at develop-

(C) 2008 The Japan Society for Aeronautical and Space Sciences ment of an environmentally friendly helicopter by focusing on noise and vibration reduction.

To achieve these goals, one of the most important parts in helicopter research is improved understanding and prediction of rotor wake aerodynamics. Compared to the fixedwing aircraft, the helicopter experiences much more complex wakes from the rotor according to the operating states and flight conditions. These complex wakes have a strong effect on vibration and noise ${ }^{3)}$ through changes in air-loads, especially when the wakes remain near the rotor-disc during descending flight for landing or maneuvering. Many different techniques have been used to analyze rotor wake more accurately, including analytical, numerical, and experimental approaches, and Ref. 4) gives a good review of previous and ongoing research on rotor wakes. However, most studies for accurate wake capturing are restricted to limited flight conditions that are relatively simple, such as hovering, level flight, or landing with steady flight condition to avoid complicity from unsteady body motion.

When considering a real-world helicopter maneuver in a complex motion, the rotor wake is much more complicated, including unsteady, 3-dimensionally-aperiodic, and transient effects as well as pitch, roll, and yaw. This fully unsteady rotor wake including tip vortex can cause significant increases in noise compared to steady flight. ${ }^{5)}$ Due to the complicity of the problems, few researchers have addressed maneuvering rotorcraft. Brentner et al. ${ }^{6)}$ conducted computational analysis using a free-vortex wake model and acoustic code to predict the unsteady loading and noise during simple maneuvers, such as turning and descending flight compared to level flight. Ananthan et al. ${ }^{7}$ predicted the transient aerodynamics of rotor wake in response to timedependent blade pitch inputs. Chen et al. ${ }^{8)}$ also examined 
three types of flight maneuvers (arrested descent, turns, and roll-reversal maneuvers) using a rotorcraft aero-acoustics prediction model. Munsky et al. ${ }^{9)}$ analyzed blade-vortex interaction (BVI) noise with flight path or attitude modification in low-speed descent. Perez et al. ${ }^{10)}$ extended the cases to other types of maneuvers using an aerodynamic/acoustic computation chain for BVI noise prediction.

Even when the overall phenomena of maneuvering rotorcraft are analyzed, the detailed characteristics of rotor wakes including tip-vortex strength and position are not still fully understood. A more accurate trajectory and the structure of the tip vortex should be taken into account for unsteady aerodynamics when noise and vibration are concerned. We have been developing our own CFD code using an accurate flow solver and acoustic solver to solve full helicopter flight. Previous research has shown the ability to capture accurate tip vortex traces in several problems. ${ }^{11,12)}$ Also the code expands the ability to solve the flow-field during interaction phenomena between each component, including tail-rotor $^{13,14)}$ and fuselage. ${ }^{15)}$ A maneuvering rotorcraft is the next important challenge on the way to full helicopter simulation.

The objectives of the present research are to construct a flow simulation (CFD) code to capture the rotor wake of a maneuvering helicopter by using new governing equations, and to apply the code to two simulation cases to understand the complex rotor wake of a maneuvering helicopter. As the first step, we propose a hybrid approach by deriving new governing equations for a maneuvering helicopter. This hybrid approach splits the motions of a maneuvering helicopter into translation, and rotation. Among all helicopter motions during maneuvering, body translation is simulated by using a translating reference frame that moves irrotationally, and all other rotation motions are simulated by moving each grid itself, including rotor rotation, blade motions (feathering, flapping, and lead-lag motions), and body rotation (pitch, roll, and yaw). Using this hybrid approach, relatively simple governing equations can be achieved compared to more complex governing equations at body-fitted coordinate frames in general motion including both translation and rotation. The CFD code is applied to simulate two typical flights: (1) accelerating/ decelerating flight, and (2) right-turn flight, by using the overlapped grid method, which is now one of the most advanced techniques for tip-vortex capture. Although it is difficult to fully validate the code due to a lack of experimental data, the validity is partly proved by discussing the relevance between a maneuvering helicopter and strong BVI noise, which is reported by other researchers. ${ }^{6,8)}$ The results of this study provide better understanding of the characteristics for a maneuvering rotorcraft, which can be valuable in full helicopter design.

\section{Governing Equations}

\subsection{Coordinate frames}

The conservation form of the Euler equations for absolute

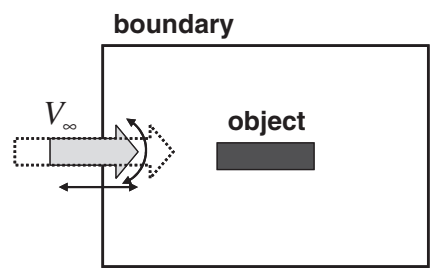

(a)

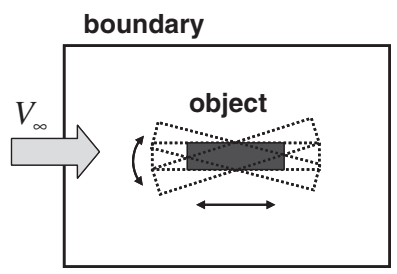

(b)
Fig. 1. Diagram of treatments for moving object.

(a) Fixed object with changing boundary condition, (b) Moving object with constant boundary condition.

motion of flow in a fixed (inertial) reference coordinate is given by:

Continuity:

$$
\frac{\partial \rho}{\partial t}+\nabla \cdot \rho \vec{V}=0
$$

Momentum:

$$
\frac{\partial \rho \vec{V}}{\partial t}+\nabla \cdot(\rho \vec{V} \vec{V}+p \vec{I})=0
$$

Energy:

$$
\frac{\partial \rho e}{\partial t}+\nabla \cdot \rho h \vec{V}=0
$$

where

$$
e=\frac{p}{\rho(\gamma-1)}+\frac{1}{2} \vec{V}^{2}
$$

and

$$
h=e+\frac{p}{\rho} .
$$

The general motion in a relative coordinate system can be implemented in two ways for the numerical solver as shown in Fig. 1 according to the treatment of the movement. For a fixed object with changing boundary conditions, the movement in the computational domain can be included numerically in the governing equations without rotation of the object axis. Alternatively, the movement can be modeled directly by moving the grid itself, i.e., moving the object with constant boundary conditions.

Until now, most CFD codes ${ }^{16)}$ for helicopters are developed for a constant-rotating blade in a constant free-stream inflow condition, considering blade motions such as feathering, flapping, and lead-lag. Then, the governing equations above are modified for coordinates fixed to a rotating rotor blade of the helicopter during hovering or level flight, which is accelerating relative to the fixed coordinates. In this case, the governing equations are expressed as follows: ${ }^{17)}$ Continuity:

$$
\frac{\partial \rho}{\partial t}+\nabla \cdot \rho \vec{V}_{\mathrm{r}}=0
$$

Momentum: 


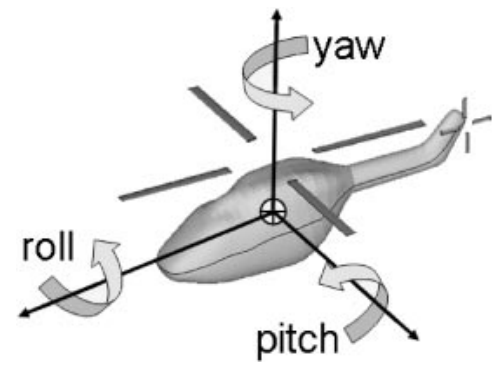

Fig. 2. General movement of helicopter

$$
\begin{aligned}
& \frac{\partial \rho \vec{V}_{\mathrm{r}}}{\partial t}+\nabla \cdot\left(\rho \vec{V}_{\mathrm{r}} \vec{V}_{\mathrm{r}}+p \overline{\bar{I}}\right) \\
& \quad=-2 \rho\left(\vec{\varpi} \times \vec{V}_{\mathrm{r}}+\vec{\varpi} \times(\vec{\varpi} \times \vec{r})\right)
\end{aligned}
$$

Energy:

$$
\frac{\partial \rho E}{\partial t}+\nabla \cdot \rho H \vec{V}_{\mathrm{r}}=0
$$

where

$$
E=e_{\mathrm{r}}-\frac{1}{2} \vec{V}_{\mathrm{t}}^{2}=\frac{p}{\rho(\gamma-1)}+\frac{1}{2} \vec{V}_{\mathrm{r}}^{2}-\frac{1}{2} \vec{V}_{\mathrm{t}}^{2}
$$

and

$$
H=E+\frac{p}{\rho}
$$

We should mention that an important presupposition for these governing equations is a constant-moving reference frame. To simulate a maneuvering helicopter, governing equations should be modified to handle both general movements such as pitching, rolling, and yawing motions as shown in Fig. 2, and also accelerating/decelerating inflow conditions according to full unsteady flight of the reference frame. Figure 3 shows diagrams of two typical methods of the moving reference frame for the helicopter problems in complex motions. The first uses a body-fitted coordinate for generally translating and a rotating reference frame as shown in Fig. 3(a). Its advantage is that the same relative position between each object of the helicopter in the reference frame simplifies grid generation, but the governing equations become too complex during coordinate transformation. ${ }^{17,18)}$ In particular, the energy equations, which should be rearranged for a rotating coordinate system, produce too many terms to implement for numerical code. The second uses a translating reference frame with rotating object as shown in Fig. 3(b). The computational domain is assumed to move irrotationally to produce relatively simple governing equations compared to the other method. The body motion (pitch, roll, and yaw) and blade rotation are simulated by moving each grid in the translating reference coordinate. Another method uses a ground-fixed coordinate frame that does not use coordinate transformation, but it must cover a huge computational domain and is considered in this paper. We chose the second method (translating reference frame with rotating object) to simulate a maneuvering helicopter. The next section derives the governing equa-

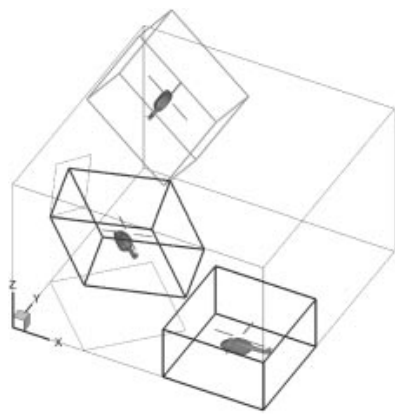

(a) General translating \& rotating reference fram.

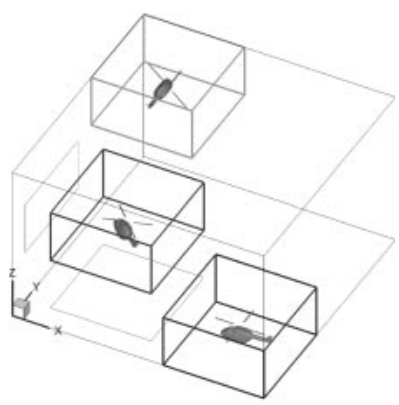

(b) Translating reference frame with rotating object.

Fig. 3. Diagrams of two different methods of moving reference frame.

tion for the translating reference frame with rotating object. For simplicity, the derivation is described by 2-dimensional equations only, but the final governing equations are written in three-dimensional form.

\subsection{Euler equations for translating reference frame in component form}

The conservation form of the Euler equations for absolute motion of flow in a fixed (inertial) reference coordinate are given by:

Continuity:

$$
\frac{\partial \rho}{\partial t}+\frac{\partial \rho u}{\partial x}+\frac{\partial \rho v}{\partial y}=0
$$

$x$-momentum:

$$
\frac{\partial \rho u}{\partial t}+\frac{\partial\left(\rho u^{2}+p\right)}{\partial x}+\frac{\partial \rho u v}{\partial y}=0
$$

$y$-momentum:

$$
\frac{\partial \rho v}{\partial t}+\frac{\partial \rho u v}{\partial x}+\frac{\partial\left(\rho v^{2}+p\right)}{\partial y}=0
$$

Energy:

$$
\frac{\partial \rho e}{\partial t}+\frac{\partial \rho h u}{\partial x}+\frac{\partial \rho h v}{\partial y}=0,
$$

where

$$
e=\frac{p}{\rho(\gamma-1)}+\frac{1}{2} \vec{V}^{2},
$$

and

$$
h=e+\frac{p}{\rho} .
$$


After coordinate transformation from inertial (fixed) coordinates into non-inertial moving (translating only) coordinates, the velocity vector can be divided as $\vec{V}=\vec{V}_{\mathrm{r}}+\vec{V}_{\mathrm{t}}$, which can be expressed in component forms as $u=u^{\prime}+$ $u_{\mathrm{t}}$ and $v=v^{\prime}+v_{\mathrm{t}}$, where $u=\partial S_{x} / \partial t, v=\partial S_{y} / \partial t, u^{\prime}=$ $\partial r_{x} / \partial t, v^{\prime}=\partial r_{y} / \partial t, u_{\mathrm{t}}=\partial R_{x} / \partial t$, and $v_{\mathrm{t}}=\partial R_{y} / \partial t$.

Among these velocities, $\vec{V}=\vec{V}(x, y, t)$ and $\vec{V}_{\mathrm{t}}=\vec{V}_{\mathrm{t}}(t)$ are defined with respect to the fixed inertial frame $(x, y)$, and $\vec{V}_{\mathrm{r}}=\vec{V}_{\mathrm{r}}\left(x^{\prime}, y^{\prime}, t^{\prime}\right)$ is defined with respect to the translating non-inertial frame $\left(x^{\prime}, y^{\prime}\right)$. After coordinate transformation from inertial (fixed) coordinates into non-inertial moving (translating only) coordinates by $u=u^{\prime}+u_{\mathrm{t}}$ and $v=v^{\prime}+$ $v_{\mathrm{t}}$, the conservation form of the Euler equations (continuity, momentum, and energy equation) can be extended as follows:

\section{Continuity:}

Using the chain rule, we can convert derivatives from the inertial frame to translating frame,

$$
\begin{aligned}
\frac{\partial}{\partial x} & =\frac{\partial}{\partial x^{\prime}}, \\
\frac{\partial}{\partial y} & =\frac{\partial}{\partial y^{\prime}}, \\
\frac{\partial}{\partial t} & =\frac{\partial}{\partial t^{\prime}}-u_{\mathrm{t}} \frac{\partial}{\partial x^{\prime}}-v_{\mathrm{t}} \frac{\partial}{\partial y^{\prime}}=\frac{\partial}{\partial t^{\prime}}-\vec{V}_{\mathrm{t}} \cdot \nabla .
\end{aligned}
$$

The conservation form of continuity is expanded as

$$
\frac{\partial \rho}{\partial t^{\prime}}-u_{\mathrm{t}} \frac{\partial \rho}{\partial x^{\prime}}-v_{\mathrm{t}} \frac{\partial \rho}{\partial y^{\prime}}+\frac{\partial \rho u}{\partial x^{\prime}}+\frac{\partial \rho v}{\partial y^{\prime}}=0
$$

By replacing $\rho=\rho^{\prime}, u=u^{\prime}+u_{\mathrm{t}}$ and $v=v^{\prime}+v_{\mathrm{t}}$, we can get

$$
\frac{\partial \rho}{\partial t^{\prime}}-u_{\mathrm{t}} \frac{\partial \rho}{\partial x^{\prime}}-v_{\mathrm{t}} \frac{\partial \rho}{\partial y^{\prime}}+\frac{\partial \rho u^{\prime}}{\partial x^{\prime}}+\frac{\partial \rho u_{\mathrm{t}}}{\partial x^{\prime}}+\frac{\partial \rho v^{\prime}}{\partial y^{\prime}}+\frac{\partial \rho v_{\mathrm{t}}}{\partial y^{\prime}}=0 .
$$

Because $\left(u_{\mathrm{t}}, v_{\mathrm{t}}\right)$ is independent of $\left(x^{\prime}, y^{\prime}\right), u_{\mathrm{t}}$ and $v_{\mathrm{t}}$ can be removed from derivative terms as

$$
\frac{\partial \rho}{\partial t^{\prime}}-u_{\mathrm{t}} \frac{\partial \rho}{\partial x^{\prime}}-v_{\mathrm{t}} \frac{\partial \rho}{\partial y^{\prime}}+\frac{\partial \rho u^{\prime}}{\partial x^{\prime}}+u_{\mathrm{t}} \frac{\partial \rho}{\partial x^{\prime}}+\frac{\partial \rho v^{\prime}}{\partial y^{\prime}}+v_{\mathrm{t}} \frac{\partial \rho}{\partial y^{\prime}}=0 .
$$

Then, the final form of the energy equation in the reference frame $\left(x^{\prime}, y^{\prime}\right)$ is:

$$
\frac{\partial \rho}{\partial t^{\prime}}+\frac{\partial \rho u^{\prime}}{\partial x^{\prime}}+\frac{\partial \rho v^{\prime}}{\partial y^{\prime}}=0
$$

which is the same form as the original continuity equation but the variables are changed for the new coordinates.

Momentum:

Similarly, the momentum equation in the $x$-direction in the $\left(x^{\prime}, y^{\prime}\right)$ frame is:

$$
\frac{\partial \rho u}{\partial t^{\prime}}-u_{\mathrm{t}} \frac{\partial \rho u}{\partial x^{\prime}}-v_{\mathrm{t}} \frac{\partial \rho u}{\partial y^{\prime}}+\frac{\partial\left(\rho u^{2}+p\right)}{\partial x^{\prime}}+\frac{\partial \rho u v}{\partial y^{\prime}}=0 .
$$

Replacing $\rho=\rho^{\prime}, u=u^{\prime}+u_{\mathrm{t}}$, and $v=v^{\prime}+v_{\mathrm{t}}$ leads to

$$
\frac{\partial \rho u^{\prime}}{\partial t^{\prime}}+\frac{\partial \rho u_{\mathrm{t}}}{\partial t^{\prime}}-u_{\mathrm{t}} \frac{\partial \rho u^{\prime}}{\partial x^{\prime}}-u_{\mathrm{t}} \frac{\partial \rho u_{\mathrm{t}}}{\partial x^{\prime}}-v_{\mathrm{t}} \frac{\partial \rho u^{\prime}}{\partial y^{\prime}}-v_{\mathrm{t}} \frac{\partial \rho u_{\mathrm{t}}}{\partial y^{\prime}}
$$

$$
\begin{aligned}
& +\frac{\partial\left(\rho u^{\prime 2}+p\right)}{\partial x^{\prime}}+2 \frac{\partial \rho u^{\prime} u_{\mathrm{t}}}{\partial x^{\prime}}+\frac{\partial \rho u_{\mathrm{t}}^{2}}{\partial x^{\prime}}+\frac{\partial \rho u^{\prime} v^{\prime}}{\partial y^{\prime}} \\
& +\frac{\partial \rho u_{\mathrm{t}} v^{\prime}}{\partial y^{\prime}}+\frac{\partial \rho u^{\prime} v_{\mathrm{t}}}{\partial y^{\prime}}+\frac{\partial \rho u_{\mathrm{t}} v_{\mathrm{t}}}{\partial y^{\prime}}=0 .
\end{aligned}
$$

Because $\left(u_{\mathrm{t}}, v_{\mathrm{t}}\right)$ is independent of $\left(x^{\prime}, y^{\prime}\right)$, we can rewrite the terms as:

$$
\begin{aligned}
& \frac{\partial \rho u^{\prime}}{\partial t^{\prime}}+\rho \frac{\partial u_{\mathrm{t}}}{\partial t^{\prime}}+u_{\mathrm{t}} \frac{\partial \rho}{\partial t^{\prime}}-u_{\mathrm{t}} \frac{\partial \rho u^{\prime}}{\partial x^{\prime}} \\
& -u_{\mathrm{t}}^{2} \frac{\partial \rho}{\partial x^{\prime}}-v_{\mathrm{t}} \frac{\partial \rho u^{\prime}}{\partial y^{\prime}}-u_{\mathrm{t}} v_{\mathrm{t}} \frac{\partial \rho}{\partial y^{\prime}} \\
& +\frac{\partial\left(\rho u^{\prime 2}+p\right)}{\partial x^{\prime}}+2 u_{\mathrm{t}} \frac{\partial \rho u^{\prime}}{\partial x^{\prime}}+u_{\mathrm{t}}^{2} \frac{\partial \rho}{\partial x^{\prime}} \\
& +\frac{\partial \rho u^{\prime} v^{\prime}}{\partial y^{\prime}}+u_{\mathrm{t}} \frac{\partial \rho v^{\prime}}{\partial y^{\prime}}+v_{\mathrm{t}} \frac{\partial \rho u^{\prime}}{\partial y^{\prime}}+u_{\mathrm{t}} v_{\mathrm{t}} \frac{\partial \rho}{\partial y^{\prime}}=0 .
\end{aligned}
$$

By deleting three pairs with opposite signs and re-arranging, we get:

$$
\begin{aligned}
& \frac{\partial \rho u^{\prime}}{\partial t^{\prime}}+\frac{\partial\left(\rho u^{\prime 2}+p\right)}{\partial x^{\prime}}+\frac{\partial \rho u^{\prime} v^{\prime}}{\partial y^{\prime}}+u_{\mathrm{t}}\left\{\frac{\partial \rho}{\partial t^{\prime}}+\frac{\partial \rho u^{\prime}}{\partial x^{\prime}}+\frac{\partial \rho v^{\prime}}{\partial y^{\prime}}\right\} \\
& =-\rho \frac{\partial u_{\mathrm{t}}}{\partial t^{\prime}}
\end{aligned}
$$

Applying continuity, $\frac{\partial \rho}{\partial t^{\prime}}+\frac{\partial \rho u^{\prime}}{\partial x^{\prime}}+\frac{\partial \rho v^{\prime}}{\partial y^{\prime}}=0$, gives the final form of the momentum equation in the $x$-direction in the reference frame $\left(x^{\prime}, y^{\prime}\right)$ as:

$$
\frac{\partial \rho u^{\prime}}{\partial t^{\prime}}+\frac{\partial\left(\rho u^{\prime 2}+p\right)}{\partial x^{\prime}}+\frac{\partial \rho u^{\prime} v^{\prime}}{\partial y^{\prime}}=-\rho \frac{\partial u_{\mathrm{t}}}{\partial t^{\prime}} .
$$

Similarly, the momentum equation in the $y$-direction can be written as:

$$
\frac{\partial \rho v^{\prime}}{\partial t^{\prime}}+\frac{\partial \rho u^{\prime} v^{\prime}}{\partial x^{\prime}}+\frac{\partial\left(\rho v^{\prime 2}+p\right)}{\partial y^{\prime}}=-\rho \frac{\partial v_{\mathrm{t}}}{\partial t^{\prime}} .
$$

\section{Energy:}

Derivation of the energy equation is comparably complex due to the kinetic energy term. Similarly, the energy equation can be expanded as:

$$
\frac{\partial \rho e}{\partial t^{\prime}}-u_{\mathrm{t}} \frac{\partial \rho e}{\partial x^{\prime}}-v_{\mathrm{t}} \frac{\partial \rho e}{\partial y^{\prime}}+\frac{\partial(\rho e+p) u}{\partial x^{\prime}}+\frac{\partial(\rho e+p) v}{\partial y^{\prime}}=0,
$$

where

$$
e=\frac{p}{\rho(\gamma-1)}+\frac{1}{2} \vec{V}^{2}
$$

and

$$
h=e+\frac{p}{\rho} .
$$

Replacing $\rho=\rho^{\prime}, u=u^{\prime}+u_{\mathrm{t}}$, and $v=v^{\prime}+v_{\mathrm{t}}$ leads to

$$
\begin{gathered}
\frac{\partial \rho e}{\partial t^{\prime}}-u_{\mathrm{t}} \frac{\partial \rho e}{\partial x^{\prime}}-v_{\mathrm{t}} \frac{\partial \rho e}{\partial y^{\prime}}+\frac{\partial(\rho e+p) u^{\prime}}{\partial x^{\prime}}+\frac{\partial(\rho e+p) u_{\mathrm{t}}}{\partial x^{\prime}} \\
+\frac{\partial(\rho e+p) v^{\prime}}{\partial y^{\prime}}+\frac{\partial(\rho e+p) v_{\mathrm{t}}}{\partial y^{\prime}}=0 .
\end{gathered}
$$


Because $\left(u_{\mathrm{t}}, v_{\mathrm{t}}\right)$ is independent of $\left(x^{\prime}, y^{\prime}\right)$, we can extract $u_{\mathrm{t}}$ and $v_{\mathrm{t}}$ as:

$$
\begin{aligned}
& \frac{\partial \rho e}{\partial t^{\prime}}-u_{\mathrm{t}} \frac{\partial \rho e}{\partial x^{\prime}}-v_{\mathrm{t}} \frac{\partial \rho e}{\partial y^{\prime}}+\frac{\partial(\rho e+p) u^{\prime}}{\partial x^{\prime}} \\
& \quad+u_{\mathrm{t}} \frac{\partial(\rho e+p)}{\partial x^{\prime}}+\frac{\partial(\rho e+p) v^{\prime}}{\partial y^{\prime}}+v_{\mathrm{t}} \frac{\partial(\rho e+p)}{\partial y^{\prime}}=0 .
\end{aligned}
$$

By deleting terms, $u_{\mathrm{t}} \frac{\partial \rho e}{\partial x^{\prime}}$ and $v_{\mathrm{t}} \frac{\partial \rho e}{\partial y^{\prime}}$, the energy equations can be written as

$$
\begin{aligned}
\frac{\partial \rho e}{\partial t^{\prime}} & +\frac{\partial(\rho e+p) u^{\prime}}{\partial x^{\prime}}+\frac{\partial(\rho e+p) v^{\prime}}{\partial y^{\prime}} \\
= & -\left\{u_{\mathrm{t}} \frac{\partial p}{\partial x^{\prime}}+v_{\mathrm{t}} \frac{\partial p}{\partial y^{\prime}}\right\},
\end{aligned}
$$

where $e=\frac{p}{\rho(\gamma-1)}+\frac{1}{2}\left(u^{2}+v^{2}\right)$, and $h=e+\frac{p}{\rho}$ are unchanged.

At this point, the energy equation can be written in different forms according to the definition of $e$ and $h$. If we want to use only relative velocities $\left(u^{\prime}, v^{\prime}\right)$ to define $e$ and $h$, we can re-arrange the terms as:

$$
\begin{aligned}
e & =\frac{p}{\rho(\gamma-1)}+\frac{1}{2}\left(u^{2}+v^{2}\right) \\
& =\frac{p}{\rho(\gamma-1)}+\frac{1}{2}\left\{\left(u^{\prime}+u_{\mathrm{t}}\right)^{2}+\left(v^{\prime}+v_{\mathrm{t}}\right)^{2}\right\} \\
& =\underbrace{\frac{p}{\rho(\gamma-1)}+\frac{1}{2}\left(u^{\prime 2}+v^{\prime 2}\right)}_{e_{\mathrm{r}}}+\underbrace{\left(u^{\prime} u_{\mathrm{t}}+v^{\prime} v_{\mathrm{t}}\right)+\frac{1}{2}\left(u_{\mathrm{t}}^{2}+v_{\mathrm{t}}^{2}\right)}_{e_{\mathrm{t}}} \\
& =e_{\mathrm{r}}+e_{\mathrm{t}},
\end{aligned}
$$

where $e_{\mathrm{r}}$ and $e_{\mathrm{t}}$ are defined as

$$
\begin{aligned}
& e_{\mathrm{r}}=\frac{p}{\rho(\gamma-1)}+\frac{1}{2}\left(u^{\prime 2}+v^{\prime 2}\right), \\
& e_{\mathrm{t}}=\left(u^{\prime} u_{\mathrm{t}}+v^{\prime} v_{\mathrm{t}}\right)+\frac{1}{2}\left(u_{\mathrm{t}}^{2}+v_{\mathrm{t}}^{2}\right) .
\end{aligned}
$$

By replacing $e=e_{\mathrm{r}}+e_{\mathrm{t}}$, the energy equation can be written as:

$$
\begin{aligned}
\frac{\partial \rho e_{\mathrm{r}}}{\partial t^{\prime}} & +\frac{\partial \rho e_{\mathrm{t}}}{\partial t^{\prime}}+\frac{\partial\left(\rho e_{\mathrm{r}}+p\right) u^{\prime}}{\partial x^{\prime}}+\frac{\partial \rho e_{\mathrm{t}} u^{\prime}}{\partial x^{\prime}} \\
+ & \frac{\partial\left(\rho e_{\mathrm{r}}+p\right) v^{\prime}}{\partial y^{\prime}}+\frac{\partial \rho e_{\mathrm{t}} v^{\prime}}{\partial y^{\prime}} \\
& =-\left\{u_{\mathrm{t}} \frac{\partial p}{\partial x^{\prime}}+v_{\mathrm{t}} \frac{\partial p}{\partial y^{\prime}}\right\}, \\
\frac{\partial \rho e_{\mathrm{r}}}{\partial t^{\prime}}+ & \frac{\partial\left(\rho e_{\mathrm{r}}+p\right) u^{\prime}}{\partial x^{\prime}}+\frac{\partial\left(\rho e_{\mathrm{r}}+p\right) v^{\prime}}{\partial y^{\prime}} \\
= & -\left\{\frac{\partial \rho e_{\mathrm{t}}}{\partial t^{\prime}}+\frac{\partial \rho e_{\mathrm{t}} u^{\prime}}{\partial x^{\prime}}+\frac{\partial \rho e_{\mathrm{t}} v^{\prime}}{\partial y^{\prime}}+u_{\mathrm{t}} \frac{\partial p}{\partial x^{\prime}}+v_{\mathrm{t}} \frac{\partial p}{\partial y^{\prime}}\right\} .
\end{aligned}
$$

The right-hand-side terms can be extended using $e_{\mathrm{t}}=\left(u^{\prime} u_{\mathrm{t}}+v^{\prime} v_{\mathrm{t}}\right)+\frac{1}{2}\left(u_{\mathrm{t}}^{2}+v_{\mathrm{t}}^{2}\right)$ as:

$$
\begin{aligned}
R H S= & \frac{\partial \rho u^{\prime} u_{\mathrm{t}}}{\partial t^{\prime}}+\frac{\partial \rho v^{\prime} v_{\mathrm{t}}}{\partial t^{\prime}}+\frac{\partial \rho \frac{1}{2}\left(u_{\mathrm{t}}^{2}+v_{\mathrm{t}}^{2}\right)}{\partial t^{\prime}} \\
& +\frac{\partial \rho u^{\prime 2} u_{\mathrm{t}}}{\partial x^{\prime}}+\frac{\partial \rho u^{\prime} v^{\prime} v_{\mathrm{t}}}{\partial x^{\prime}}+\frac{\partial \rho u^{\prime} \frac{1}{2}\left(u_{\mathrm{t}}^{2}+v_{\mathrm{t}}^{2}\right)}{\partial x^{\prime}} \\
& +\frac{\partial \rho v^{\prime} u^{\prime} u_{\mathrm{t}}}{\partial y^{\prime}}+\frac{\partial \rho v^{\prime 2} v_{\mathrm{t}}}{\partial y^{\prime}}+\frac{\partial \rho v^{\prime} \frac{1}{2}\left(u_{\mathrm{t}}^{2}+v_{\mathrm{t}}^{2}\right)}{\partial y^{\prime}} \\
& +u_{\mathrm{t}} \frac{\partial p}{\partial x^{\prime}}+v_{\mathrm{t}} \frac{\partial p}{\partial y^{\prime}} \cdot
\end{aligned}
$$

Because $\left(u_{\mathrm{t}}, v_{\mathrm{t}}\right)$ is independent of $\left(x^{\prime}, y^{\prime}\right)$, re-arranging the terms gives:

$$
\begin{aligned}
R H S= & \rho u^{\prime} \frac{\partial u_{\mathrm{t}}}{\partial t^{\prime}}+\rho v^{\prime} \frac{\partial v_{\mathrm{t}}}{\partial t^{\prime}}+\underbrace{\rho \frac{1}{2}\left(u_{\mathrm{t}}^{2}+v_{\mathrm{t}}^{2}\right)}_{(I V)} \\
& +u_{\mathrm{t}} \frac{\partial \rho u^{\prime}}{\partial t^{\prime}}+v_{\mathrm{t}} \frac{\partial \rho v^{\prime}}{\partial t^{\prime}}+\frac{1}{2}\left(u_{\mathrm{t}}^{2}+v_{\mathrm{t}}^{2}\right) \frac{\partial \rho}{\partial t^{\prime}} \\
& +u_{\mathrm{t}} \frac{\partial \rho u^{\prime 2}}{\partial x^{\prime}}+v_{\mathrm{t}} \frac{\partial \rho u^{\prime} v^{\prime}}{\partial x^{\prime}}+\frac{1}{2}\left(u_{\mathrm{t}}^{2}+v_{\mathrm{t}}^{2}\right) \frac{\partial \rho u^{\prime}}{\partial x^{\prime}} \\
& +u_{\mathrm{t}} \frac{\partial \rho v^{\prime} u^{\prime}}{\partial y^{\prime}}+v_{\mathrm{t}} \frac{\partial \rho v^{\prime 2}}{\partial y^{\prime}}+\frac{1}{2}\left(u_{\mathrm{t}}^{2}+v_{\mathrm{t}}^{2}\right) \frac{\partial \rho v^{\prime}}{\partial y^{\prime}} \\
& +\underbrace{u_{\mathrm{t}} \frac{\partial p}{\partial x^{\prime}}}_{(I)}+\underbrace{v_{\mathrm{t}} \frac{\partial p}{\partial y^{\prime}}}_{(I I)}
\end{aligned}
$$

By applying the continuity and momentum equations to the groups (I), (II), and (III),

$$
\begin{aligned}
(I) & =u_{\mathrm{t}} \frac{\partial \rho u^{\prime}}{\partial t^{\prime}}+u_{\mathrm{t}} \frac{\partial \rho u^{\prime 2}}{\partial x^{\prime}}+u_{\mathrm{t}} \frac{\partial p}{\partial x^{\prime}}+u_{\mathrm{t}} \frac{\partial \rho v^{\prime} u^{\prime}}{\partial y^{\prime}} \\
& =u_{\mathrm{t}}\left\{\frac{\partial \rho u^{\prime}}{\partial t^{\prime}}+\frac{\partial\left(\rho u^{\prime 2}+p\right)}{\partial x^{\prime}}+\frac{\partial \rho u^{\prime} v^{\prime}}{\partial y^{\prime}}\right\} \\
& =-\rho u_{\mathrm{t}} \frac{\partial u_{\mathrm{t}}}{\partial t^{\prime}}=-\rho \frac{\partial \frac{1}{2} u_{\mathrm{t}}^{2}}{\partial t^{\prime}}, \\
(I I)= & v_{\mathrm{t}} \frac{\partial \rho v^{\prime}}{\partial t^{\prime}}+v_{\mathrm{t}} \frac{\partial \rho u^{\prime} v^{\prime}}{\partial x^{\prime}}+v_{\mathrm{t}} \frac{\partial \rho v^{\prime 2}}{\partial y^{\prime}}+v_{\mathrm{t}} \frac{\partial p}{\partial y^{\prime}} \\
= & v_{\mathrm{t}}\left\{\frac{\partial \rho v^{\prime}}{\partial t^{\prime}}+\frac{\partial \rho u^{\prime} v^{\prime}}{\partial x^{\prime}}+\frac{\partial\left(\rho v^{\prime 2}+p\right)}{\partial y^{\prime}}\right\} \\
= & -\rho v_{\mathrm{t}} \frac{\partial v_{\mathrm{t}}}{\partial t^{\prime}}=-\rho \frac{1}{\partial t^{\prime}}, \\
(I I I)= & \frac{1}{2}\left(u_{\mathrm{t}}^{2}+v_{\mathrm{t}}^{2}\right) \frac{\partial \rho}{\partial t^{\prime}}+\frac{1}{2}\left(u_{\mathrm{t}}^{2}+v_{\mathrm{t}}^{2}\right) \frac{\partial \rho u^{\prime}}{\partial x^{\prime}} \\
& +\frac{1}{2}\left(u_{\mathrm{t}}^{2}+v_{\mathrm{t}}^{2}\right) \frac{\partial \rho v^{\prime}}{\partial y^{\prime}} \\
= & \frac{1}{2}\left(u_{\mathrm{t}}^{2}+v_{\mathrm{t}}^{2}\right)\left\{\frac{\partial \rho}{\partial t^{\prime}}+\frac{\partial \rho u^{\prime}}{\partial x^{\prime}}+\frac{\partial \rho v^{\prime}}{\partial y^{\prime}}\right\}=0 .
\end{aligned}
$$


Also (I) and (II) cancel (IV), so the final form of energy equation is:

$$
\begin{gathered}
\frac{\partial \rho e_{\mathrm{r}}}{\partial t^{\prime}}+\frac{\partial\left(\rho e_{\mathrm{r}}+p\right) u^{\prime}}{\partial x^{\prime}}+\frac{\partial\left(\rho e_{\mathrm{r}}+p\right) v^{\prime}}{\partial y^{\prime}} \\
=-\left\{\rho u^{\prime} \frac{\partial u_{\mathrm{t}}}{\partial t^{\prime}}+\rho v^{\prime} \frac{\partial v_{\mathrm{t}}}{\partial t^{\prime}}\right\},
\end{gathered}
$$

where

$$
e_{\mathrm{r}}=\frac{p}{\rho(\gamma-1)}+\frac{1}{2}\left(u^{\prime 2}+v^{\prime 2}\right)
$$

The three-dimensional governing equations are derived similarly.

$$
\begin{aligned}
& \frac{\partial^{\prime} \rho}{\partial t^{\prime}}+\nabla \cdot \rho \vec{V}_{\mathrm{r}}=0, \\
& \frac{\partial^{\prime} \rho \vec{V}_{\mathrm{r}}}{\partial t^{\prime}}+\nabla \cdot\left(\rho \vec{V}_{\mathrm{r}} \vec{V}_{\mathrm{r}}+p \vec{I}\right)=-\left(\rho \frac{\partial^{\prime} \vec{V}_{\mathrm{t}}}{\partial t^{\prime}}\right), \\
& \frac{\partial^{\prime} \rho e_{\mathrm{r}}}{\partial t^{\prime}}+\nabla \cdot \rho h_{\mathrm{r}} \vec{V}_{\mathrm{r}}=-\left\{\rho \vec{V}_{\mathrm{r}} \cdot \frac{\partial^{\prime} \vec{V}_{\mathrm{t}}}{\partial t^{\prime}}\right\},
\end{aligned}
$$

where

$$
e_{\mathrm{r}}=\frac{p}{\rho(\gamma-1)}+\frac{1}{2} \vec{V}_{\mathrm{r}}^{2},
$$

and

$$
h_{\mathrm{r}}=e_{\mathrm{r}}+\frac{p}{\rho}
$$

The final form is very simple excluding two changes from the original Euler equations: (1) relative velocities are used, and (2) source terms are added for the momentum equations and energy equation. The free-stream boundary condition should be prescribed properly according to the movement of the computational domain at each time step. The merit of these governing equations is the absence of complex source terms during code implementation.

\section{Calculation Method}

\subsection{Grid system}

We used a moving overlapped grid system with three different types of grids (helicopter grid, inner and outer background grids) as shown in Fig. 4. The helicopter grid consists of many grid blocks matching the helicopter configuration to simulate blades of the main-/tail-rotor, fuselage, etc. The inner background grid is placed around the rotor disk. The outer background grid covers the whole computation region with a sparse grid density. The flow data are exchanged between the inner and outer background grids, and between the blade grid and the inner-background grid. The body-fitted blade grid in $\mathrm{O}-\mathrm{H}$ topology moves along with the blade motion including rotation, flapping, feathering, and lagging. Elastic motion of the blade is neglected because blades with a rigid body are considered here.

Table 1 shows the specification of each grid. Most grid

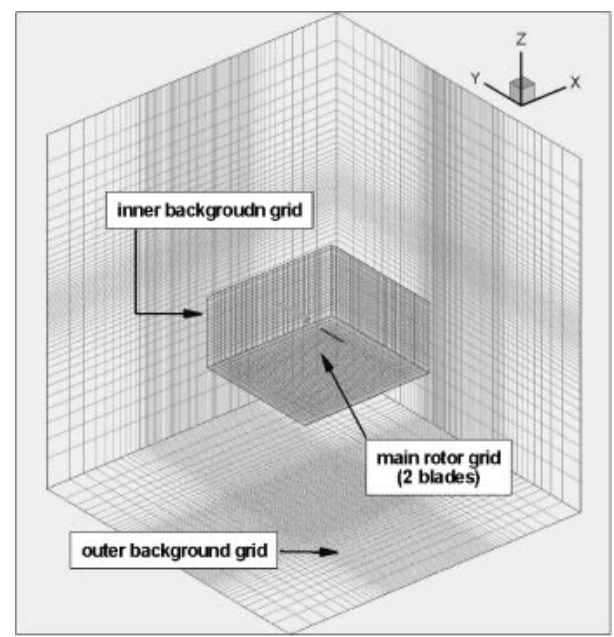

(a) Perspective view.

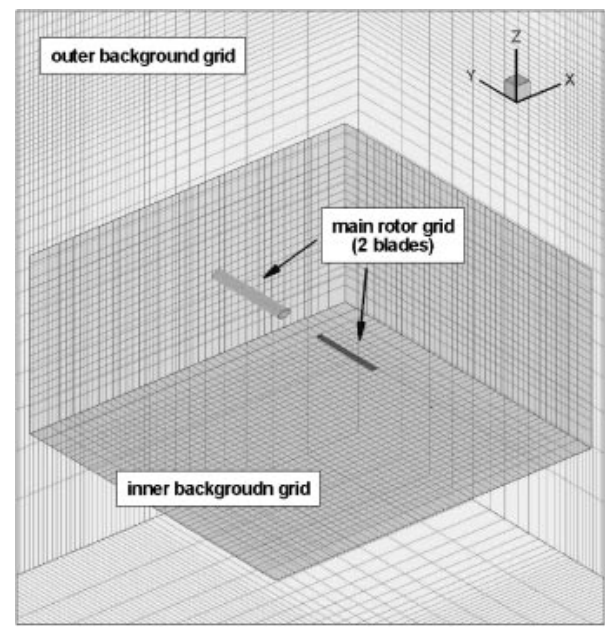

(b) Close-up of inner background grid.

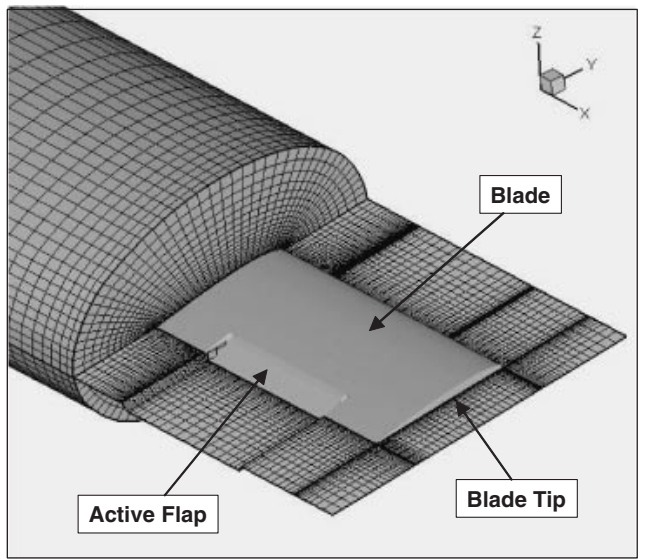

(c) Close-up of blade grid.

Fig. 4. Grid system

points are concentrated in the inner-background grid, which captures the trace of the tip vortex during several rotations. The number of grid points in the span-wise direction of the blade grid is increased considerably to model the active flap. The grid spacing of the inner background grid corresponds 
Table 1. Specification of grid system.

\begin{tabular}{cc}
\hline & Grid \\
\hline $\begin{array}{c}\text { Inner } \\
\text { background grid }\end{array}$ & $\begin{array}{c}(X \times Y \times Z) \\
\text { Outer background } \\
\text { grid }\end{array}$ \\
$\begin{array}{cc}(X \times Y \times Z) \\
\text { Blade grid }\end{array}$ & $83 \times 79 \times 49=321,293$ \\
\hline Total & $(141 \times 25 \times 125) \times 4=1,706,100$ \\
\hline $\begin{array}{c}\text { Spacing of inner } \\
\text { background grid }\end{array}$ & $0.08 c(=0.0105 R)$ \\
\hline
\end{tabular}

to $0.08 c$, where $c$ is the blade chord length. Although this grid spacing, which is specified similarly to other research works, ${ }^{19,20)}$ may not be sufficiently to capture the tip vortex accurately because of computing cost limitations, it can be sufficient for the present research objectives to compare the quantitative characteristics of a maneuvering helicopter using the new governing equations.

\subsection{Numerical schemes}

We used a three-dimensional unsteady flow solver ${ }^{21)}$ for the Euler equation to analyze the active flap aerodynamics. In the calculation of the blade grid, inviscid flux vectors are separated using Roe's flux difference splitting (FDS) algorithm, where flux difference across a cell interface is divided into the components associated with each characteristic wave with second-order accuracy using a TVD scheme, which is known to be good for capturing shock waves without adding artificial dissipation. Since Roe's approximate Riemann solver does not have consistency with the entropy condition and thus permits physically inadmissible expansion shock, an entropy correction is applied to resolve this inconvenience. For time integration, we used a first-order Euler backward scheme in the conventional delta form and time accuracy is improved by adding the Newton iteration. Four iterations are used at each time step and the typical dividing number along the azimuthal direction is about 4800 per revolution, which corresponds to an azimuth angle of about $0.075^{\circ}$. A diagonalized ADI method with an upwind flux-split technique is used in the linearized implicit part for the discretized governing equations.

In the calculations of background grids, the flux difference across cell interface is divided using a compact TVD scheme. $^{22)}$ The MUSCL cell interface value is modified to achieve 4th-order high accuracy in the background Cartesian grids. The Simple High-resolution Upwind Scheme (SHUS) ${ }^{23)}$ is used to obtain the numerical flux. SHUS is an Advection Upstream Splitting Method (AUSM) type approximate Riemann solver and has small numerical diffusion. The four-stage Runge-Kutta method is used for time integration. The free stream condition is applied for the outer boundary of the outer background grid.

In this study, calculations are performed using the Central Numerical Simulation System (CeNSS), the main part of the third-generation numerical simulator of JAXA. It is composed of high-performance UNIX servers (FUJITSU PRIMEPOWER) connected by a crossbar network. CeNSS has 9TFLOPS peak performance, $3 \mathrm{~TB}$ of RAM, $50 \mathrm{~TB}$ of disk storage, and a 600-TB tape archive. It takes about 25 hours to obtain one revolution solution for a rotor Euler calculation with about 16 million grid points using 36 CPUs. For the steady forward flight solution which is used as a starting stage, four rotor revolutions are needed to get a fully converged solution, and then more revolutions for transient motion begin according to the flight conditions.

\section{Calculation Results}

The governing equations derived in the previous section were implemented in the present CFD code, and two simple cases for maneuvering helicopter were calculated. As a result, the unsteady movement of the complex tip vortex is shown and discussed. The first case is accelerating/decelerating flight with varying forward flight velocity (i.e. varying inflow free-stream velocity). The case can demonstrate the effect of change in forward flight velocity on the tip vortex trace. The second case is an aggressive right-turn flight, which is simulated by changing the flight direction and yaw angle. The case can demonstrate the effect of flight direction on the tip vortex trace. These kinds of aggressive changes occur in the flight of military helicopters, where excessive noise arises as a critical problem. From the limits of the present computation resources (especially computing time), the transitions occur during relatively short terms: 3 rotor revolutions for the acceleration/deceleration case, and 5 rotor revolutions for the right-turn case. The blade motion data and helicopter flight parameters are given as prescribed input data. ${ }^{3)}$ The specifications for helicopter motions and flow conditions are shown in Table 2. The transitions from one stage to another stage are simulated using intermediate motion data, which are interpolated curvilinearly from the steady flight conditions. Although the present flight conditions are rather overstated, the quantitative characteristics of a maneuvering helicopter can be simulated using the new governing equations.

\subsection{Accelerating/decelerating flight}

A transient flight between forward flight and hovering flight is simulated to demonstrate the unsteady flow characteristics with acceleration and deceleration of a maneuvering helicopter. The list of flight conditions along with relevant flight state information is shown in Table 2(a).

The first transition of deceleration from level flight to the hovering flight condition occurs for 3 revolutions, then hovering flight continues for another 3 revolutions. The second transition of acceleration to recover level flight from hovering flight also occurs for 3 revolutions, then the level flight condition is continued. The conditions during transient flight are interpolated from the level flight and hovering flight for each time step. Figure 5 shows side and top views of the vorticity iso-surface at each stage. The difference in tip vortex movement due to downwash is well shown 
Table 2. Specification of helicopter motions and flight conditions.

\begin{tabular}{|c|c|c|c|c|c|c|c|c|}
\hline Case 1 & Condition & $M_{\infty}$ & $\alpha_{\mathrm{tpp}}$ & $\begin{array}{c}\text { Collective } \\
\text { (deg) }\end{array}$ & $\begin{array}{l}\text { Lateral } \\
(\mathrm{deg})\end{array}$ & $\begin{array}{l}\text { Longitudinal } \\
\text { (deg) }\end{array}$ & $\begin{array}{l}\text { Yaw } \\
(\text { deg) }\end{array}$ & $\begin{array}{l}\text { Roll } \\
\text { (deg) }\end{array}$ \\
\hline Forward & periodic & 0.109 & 3 & 5.2 & -1.33 & 2.72 & 0 & 0 \\
\hline Transient flight & aperiodic & \multicolumn{7}{|c|}{ curvilinear interpolation using flight data } \\
\hline Hovering flight & periodic & 0 & 0 & 9.92 & 0 & 0 & 0 & 0 \\
\hline Transient flight & aperiodic & \multicolumn{7}{|c|}{ curvilinear interpolation using flight data } \\
\hline Forward & periodic & 0.109 & 3 & 5.2 & -1.33 & 2.72 & 0 & 0 \\
\hline
\end{tabular}

(b) Case 2: Right-turn flight.

\begin{tabular}{ccccccccc}
\hline Case 1 & Condition & $M_{\infty}$ & $\alpha_{\mathrm{tpp}}$ & $\begin{array}{c}\text { Collective } \\
(\mathrm{deg})\end{array}$ & $\begin{array}{c}\text { Lateral } \\
(\mathrm{deg})\end{array}$ & $\begin{array}{c}\text { Longitudinal } \\
(\mathrm{deg})\end{array}$ & $\begin{array}{c}\text { Yaw } \\
(\mathrm{deg})\end{array}$ & $\begin{array}{c}\text { Roll } \\
(\mathrm{deg})\end{array}$ \\
\hline Forward & periodic & 0.109 & 3 & 5.2 & -1.33 & 2.72 & 0 & 0 \\
Transient flight & aperiodic & \multicolumn{7}{c}{ curvilinear interpolation } \\
Right-turn & periodic & 0.109 & 2.21 & 14.03 & -3.31 & 10.23 \\
Transient flight & $\begin{array}{c}\text { aperiodic } \\
\text { Forward }\end{array}$ & periodic & 0.109 & 3 & 5.2 & -1.33 & -90 & 45 \\
\hline
\end{tabular}

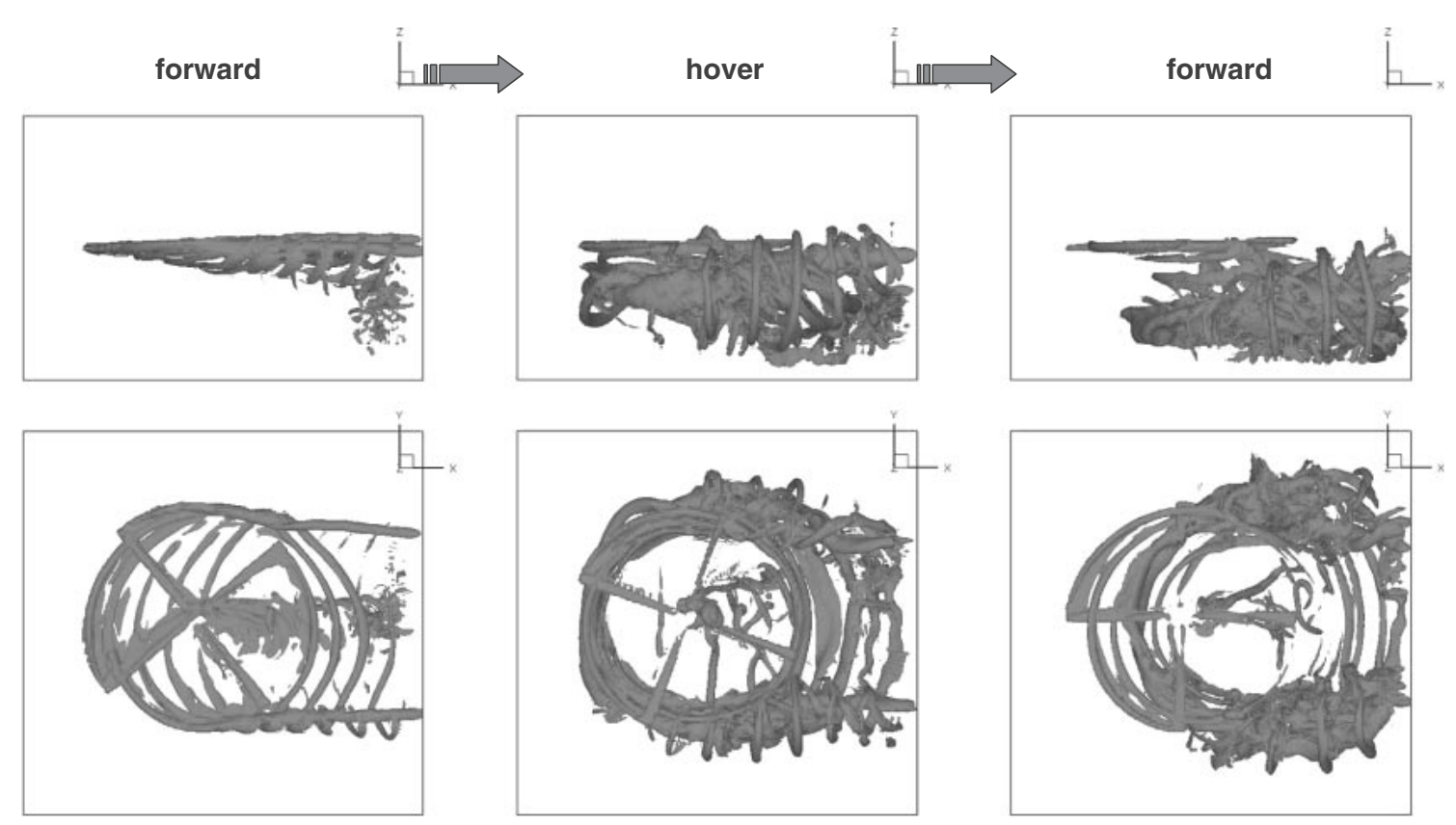

Fig. 5. Side view and top view of typical iso-surface of vorticity for accelerating/decelerating flight.

in side view figures according to the flight conditions. The top views show the effect of flight speed (in other words, inflow velocity into computational domain) with the changing stream-wise distance between tip vortices at each stage.

The tip vortices interact with each other to produce strong vibration and noise through the changes of air-loads on blade surface during interactions. Vorticity contours on sectional planes can also describe the interaction phenomena effectively. Figure 6 shows vorticity contours at one sectional plane about $63 \%$ of rotor radius in advancing side. The contours show the trace of tip vortex according to flight stages well. As deceleration begins at 4 th revolution, the downwash becomes dominant relatively because the flight speed decreases. As re-acceleration begins at 7 th revolution, the down-going tip vortex starts moving again along the stream, and the old tip vortex under the rotor disk move together with the newly-generated tip vortex as the flight speed increases. Finally the trace of tip vortex recovers its shape to be initial level flight condition.

\subsection{Right-turn flight}

A transient flight from level flight to a coordinate rightturn flight is simulated. A list of flight conditions along with relevant flight state information is shown in Table 2(b). The right-turn transition occurs for 5 revolutions including both yawing and rolling motion. After finishing the turn as the roll angle becomes $45^{\circ}$, the transition to level flight occurs for another 3 revolutions to recover the roll angle, and then the level flight is continued. The conditions during transient flight are interpolated for each time step. Figure 7 shows the 

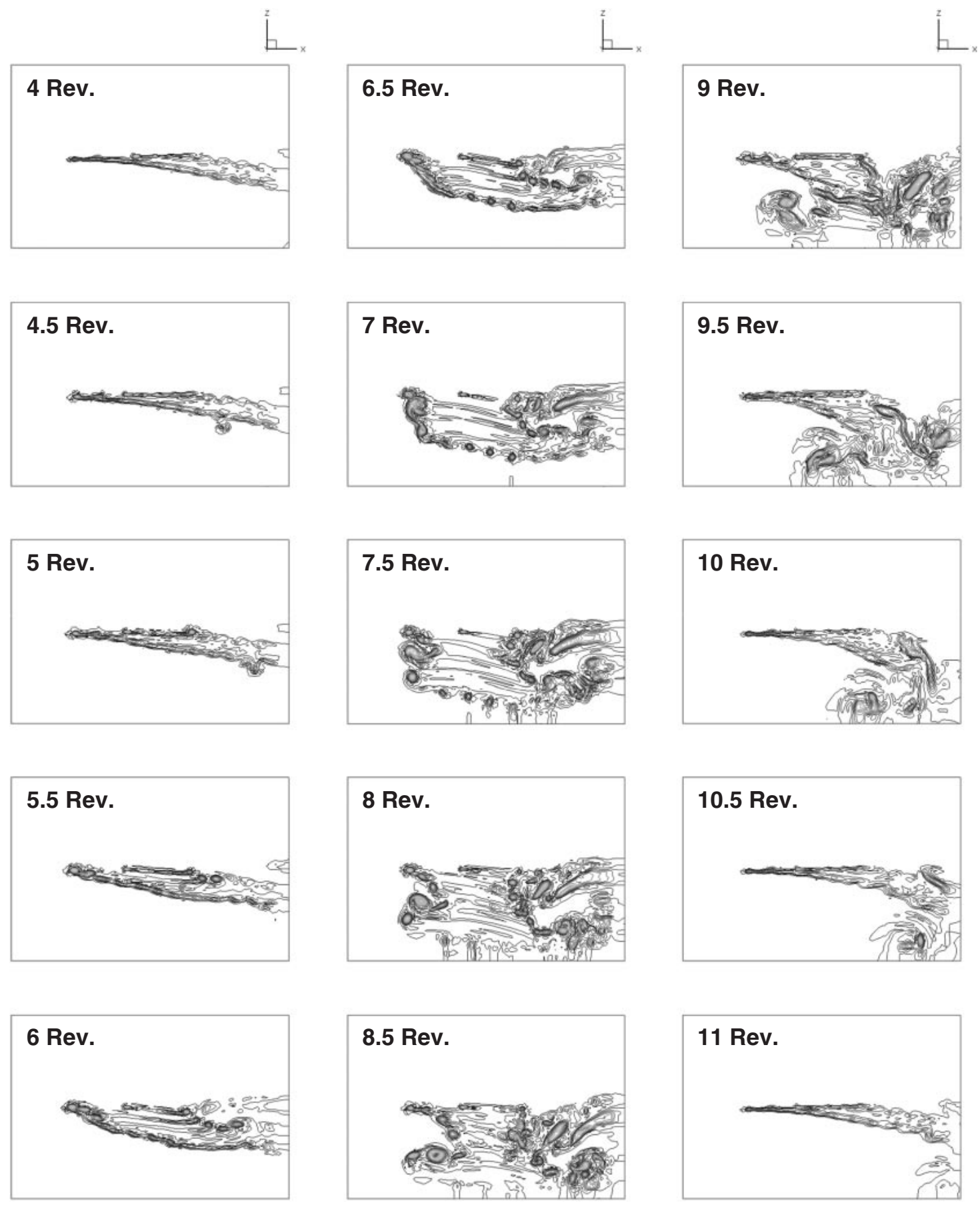

Fig. 6. Side view vorticity contour at sectional plane about $63 \%$ of rotor radius in advancing side in time.

side and top views of the vorticity iso-surface at each stage. The side views show the difference in tip vortex movement with roll angle during the right-turn. The top views show the effect of flight speed (in other words, inflow velocity to the computational domain) and flight direction (in other words, yaw angle) on the trace of the tip vortex for the flight stages. The main characteristic of turning, as shown in these figures, is that the tip vortices of the advancing side overlap to accumulate according to the rotation of helicopter itself. The blade is expected to experience stronger interactions during passage through the accumulated strong tip vortex in comparison to the ordinary blade-vortex interaction.
An advantage of using CFD is being able to closely inspect specified sectional planes to analyze rotor wake including tip vortices, which is very difficult in wind-tunnel tests and almost impossible in flight tests. From the huge amount of calculation data, we can extract the information needed to understand the detailed tip vortex behavior such as roll-up and merging. The vortex-merging phenomenon in the present right-turning flight is explained more clearly by the CFD results. Figures 8 and 9 show the vorticity contours on several stream-wise sectional planes to compare the vortex behaviors. For the level flight shown in Fig. 8, the contours at sections at $d=0.5 R, 0.75 R$, and $1.0 R$, where 


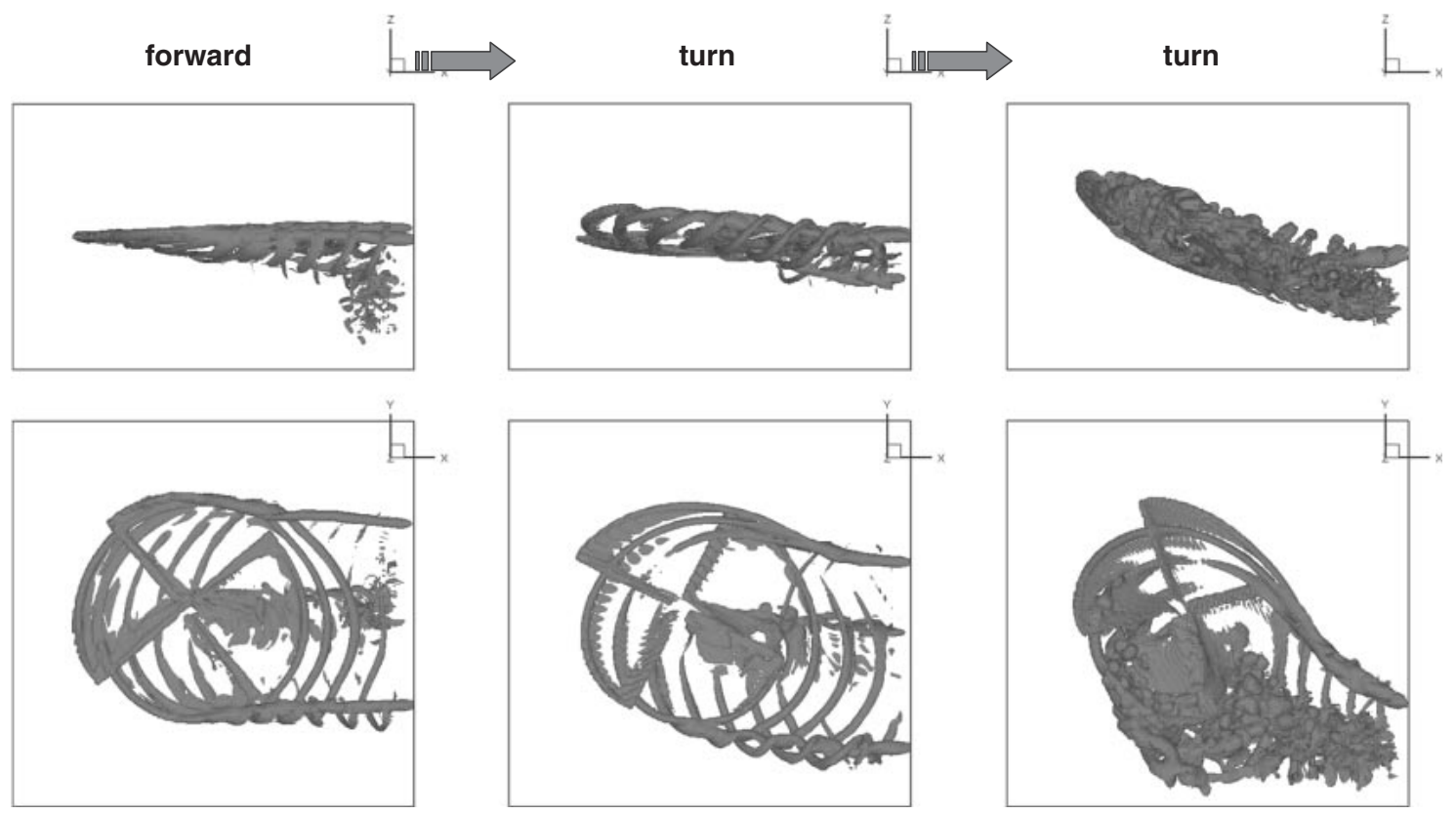

Fig. 7. Side view and top view of typical iso-surface of vorticity for right-turn flight.
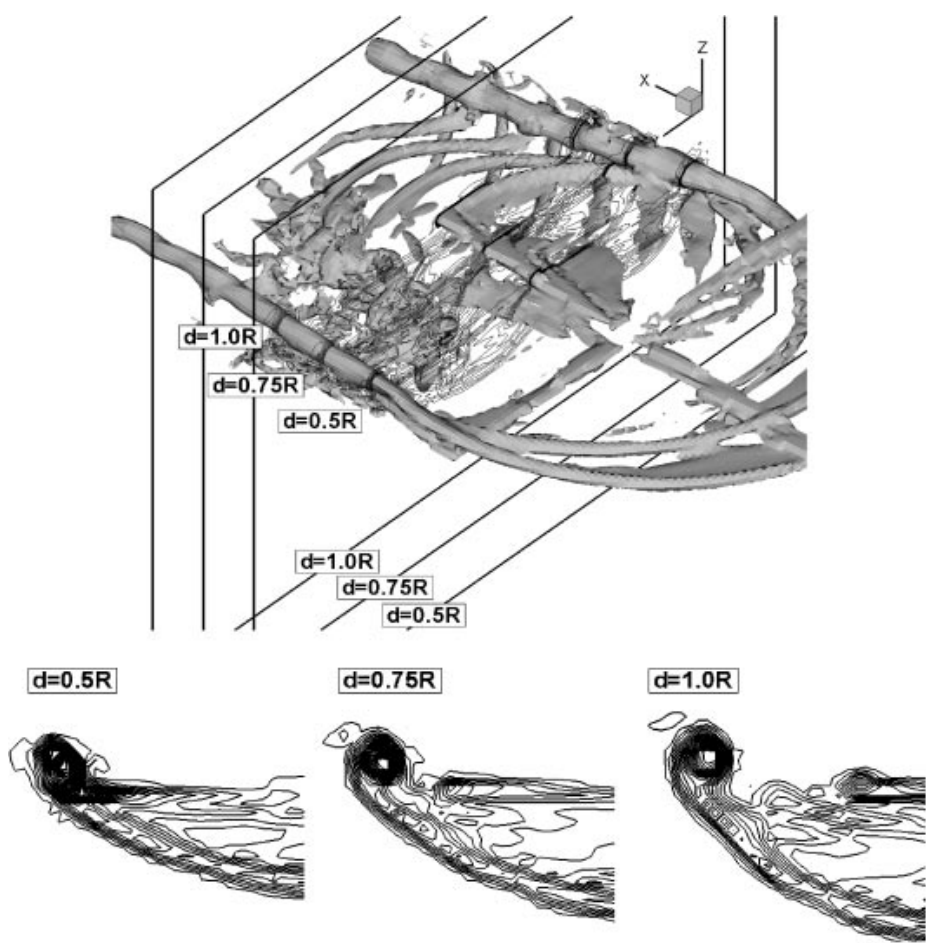

Fig. 8. Iso-surface with perspective view and vorticity contours in sectional planes during level flight.

$d$ is the downward distance from the center of rotation, show the typical pattern of the tip vortex from the advancing-side rotor. On the other hand, tip vortices in right-turn flight show quite a different pattern in Fig. 9. First, the trace of the tip vortices shows a left-biased curve owing to the right-turn flight. When drawn at several sections at $d=0.5 R, 0.75 R$, $1.0 R$ and $1.25 R$, the center of tip vortices seems to move inward, i.e., to the blade root. In fact, at the start of right-turn flight, the helicopter itself accelerates to the right, and moves close to the old tip vortex faster, which appears as the inward movement of the tip vortex at the coordinate fixed to the maneuvering helicopter. Another important characteristic of right-turn flight is a vortex bundling phenomena as vortices grow downward. This strong vortex may cause control instability and also strong vibration and noise mentioned as 'super BVI' by Brentner.5)

Figure 10 shows the unsteady pressure history at a surface point of $3 \%$ chord and $90 \%$ span for several revolutions during right-turn flight. The time derivative of pressure at this point is known to have good correlation with noise 

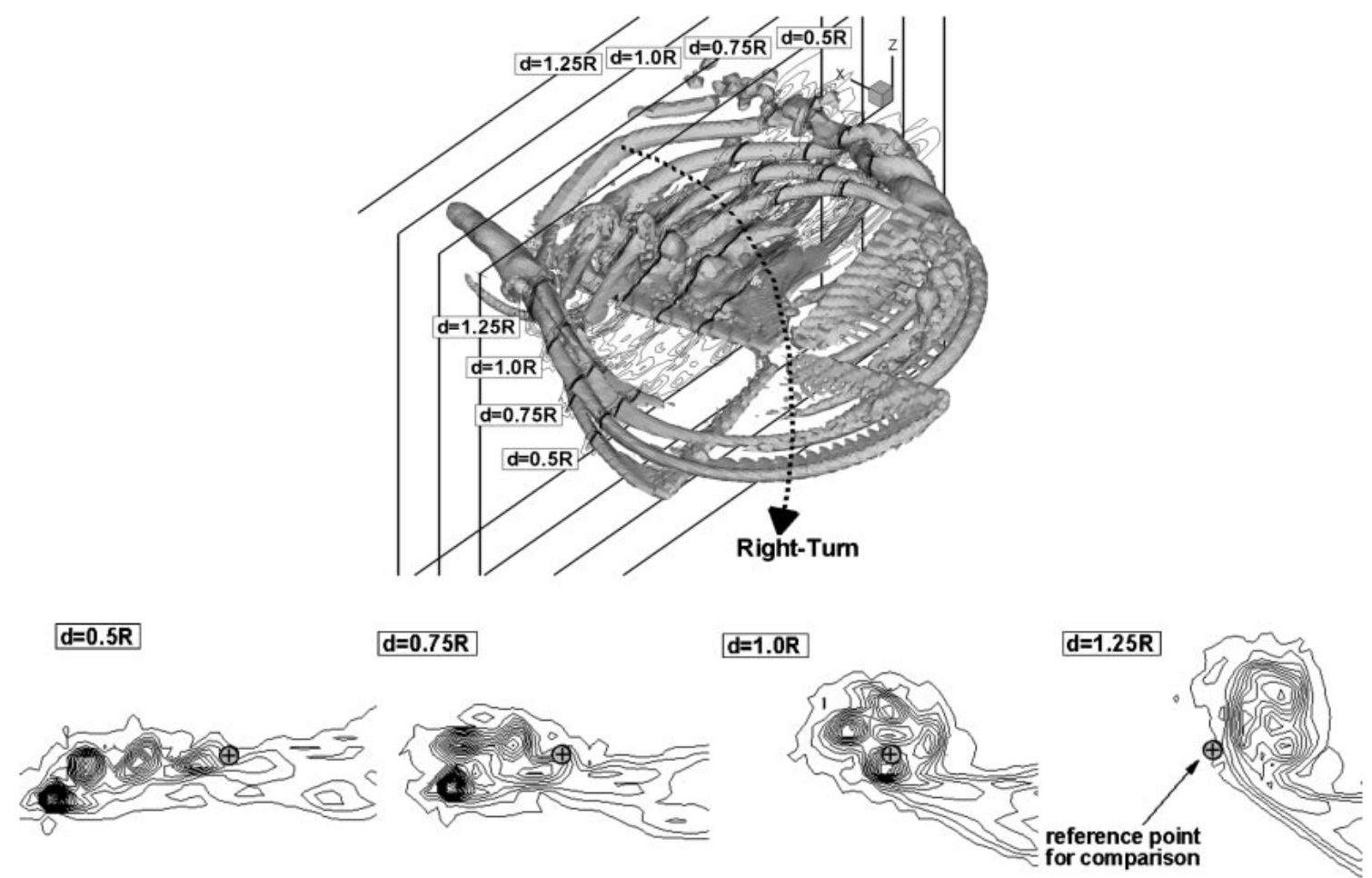

Fig. 9. Iso-surface with perspective view and vorticity contours in sectional planes during right-turn flight.
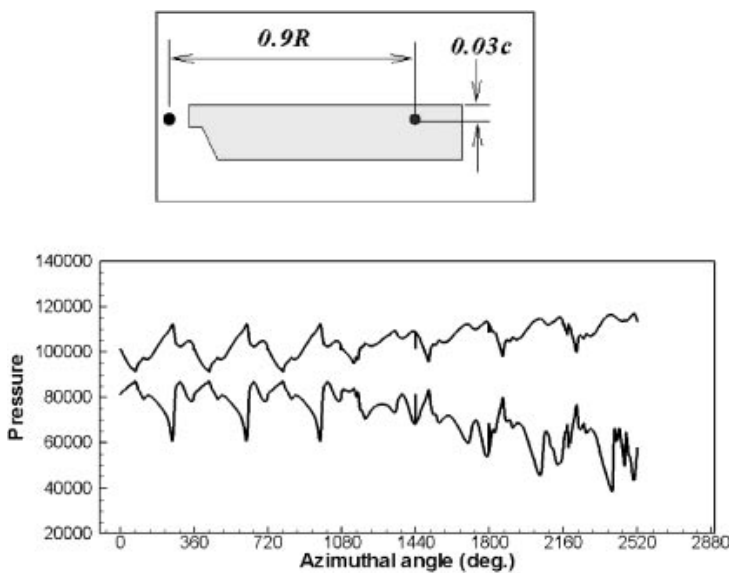

Fig. 10. Histories of pressure at point on upper/lower surface of blade.

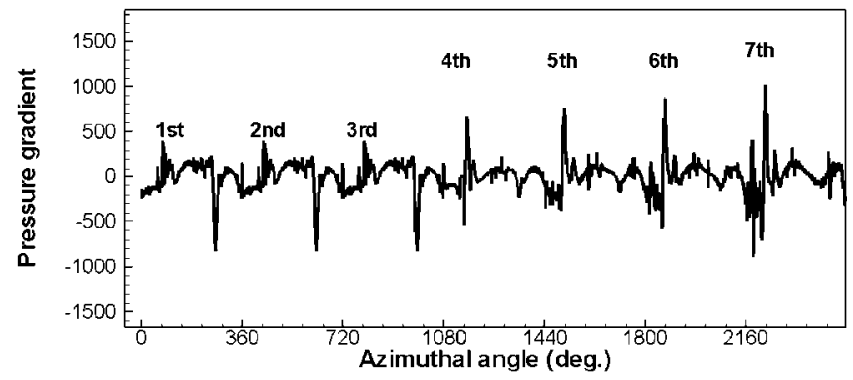

Fig. 11. Histories of pressure gradient at point on lower blade surface.

intensity. The pressure history shape changes a lot with flight conditions, especially when entering a right-turn. The effects of these sudden pressure changes are well shown by the pressure gradient history at a point on the lower blade surface in Fig. 11. The pressure gradient shows quite strong positive peaks compared to the typical BVI case. This enlarged pressure gradient, which is directly related to noise, is believed to originate from the strong interaction between the proceeding blade and the accumulated tip vortex caused by vortex bundling during a right-turn. Compared to the first three positive peaks (1st-3rd), those are thought to come from BVI in level flight, the following peaks (4th7 th) are thought to come from BVI of right-turn flight. The validity of the present CFD code for a maneuvering helicopter is partially proven by this noise pattern during right-turn flight.

\section{Summary and Conclusions}

A three-dimensional unsteady Euler code has been developed using the overlapped grid method for analysis of a maneuvering helicopter. The following results were found.

1. A hybrid approach is proposed by splitting the maneuvering motion into translation and rotation with different treatments. Translation is simulated using a translating reference frame, and rotations are simulated by moving each grid in the frame. New governing equations are derived in this non-inertial moving (translating) coordinate for the hybrid approach.

2. A flow simulation (CFD) code is constructed using the hybrid approach to capture the rotor wake of a maneuvering helicopter using an overlapped grid method, and by applying the CFD code to two simulation cases (accelerating/decelerating flight and right-turn flight). The complex phenomena of rotor wake from a maneuvering helicopter are well described using this code.

3. Vortex bundling, which is a main characteristic of 
right-turn flight, is well captured by the simulation code, and reproduction of the strong pressure gradient, which is known to be related to strong noise in rightturn flight, validates the CFD code.

\section{References}

1) http://www.theorator.com/bills108/s309.html

2) http://www.eurocopter.com/jrotor/69/friendcopter.html

3) Caradonna, F. X., Kitaplioglu, C., McCluer, M., Baeder, J., Leishman, G., Berezin, C., Visintainer, J., Bridgeman, J., Burley, C., Epstein, R., Lyrintzis, A., Koutsavdis, E., Rahrier, G., Delrieux, Y., Rule, J. and Bliss, D.: A Review of Methods for the Prediction of BVI Noise, AHS Technical Specialists Meeting for Rotorcraft Acoustics and Aerodynamics, Williamsburg, VA, October 1997.

4) Leishman, J. G., Bhagwat, M. J. and Bagai, A.: Free-Vortex Methods for Helicopter Rotor Wake Analyses, Special Edition on Rotorcraft Wakes, J. Aircraft, 39, 5 (2002), 759-775.

5) Brentner, K. S. and Jones, H. E.: Noise Prediction for Maneuvering Rotorcraft, AIAA Paper 2000-2031, 2000.

6) Brentner, K. S., Perea, G., Bres, G. A. and Jones, H. E.: Toward a Better Understanding of Maneuvering Rotorcraft Noise, AHS 58th Annual Forum, Montreal, Canada, June 2002.

7) Ananthan, S. and Leishman, J. G.: Predictions of Transient Rotor Wake Aerodynamics in Response to Time-Dependent Blade Pitch Inputs, AHS 59th Annual Forum, Phoenix, AZ, May 2003.

8) Chen, H., Brentner, K. S., Ananthan, S. and Leishman, J. G.: A Computational Study of Helicopter Rotor Wakes and Noise Generated during Transient Maneuver, AHS 61st Annual Forum, Grapevine, TX, May 2005.

9) Munsky, B., Gandhi, F. and Tauszig, L.: An Analysis of Helicoter Blade-Vortex Interaction Noise with Flight Path or Attitude Modification, AHS 58th Annual Forum, Montreal, Canada, June 2002.

10) Perez, G. and Costes, M.: A New Aerodynamic \& Acoustic Computation Chain for BVI Noise Prediction in Unsteady Flight Conditions, AHS 60th Annual Forum, Baltimore, USA, June 2004.

11) Yang, C., Aoyama, T. and Saito, S.: Numerical Study on BVI Noise Reduction Using Active Flap Control, 31st ERF, No. 24, Florence, Italy, September 2005.

12) Aoyama, T., Yang, C. and Saito, S.: Numerical Analysis of Active Flap for Noise Reduction Using Moving Overlapped Grid Method, J. Am. Helicopter Soc., 52, 3 (2007), pp. 189-200.

13) Aoyama, T., Yang, C., Kondo, N. and Saito, S.: Fundamental CFD Analysis on Main-Rotor/Tail-Rotor Interaction Noise of Helicopters, Theor. Appl. Mech. Jpn., 53 (2004), pp. 215-220.

14) Yang, C., Aoyama, T. and Saito, S.: Numerical Analysis of Interaction Noise between Main Rotor and Tail Rotor of Helicopter, 24th International Congress of the Aeronautical Science (ICAS), Yokohama, Japan, 29 Aug.-3 September 2004.

15) Yang, C., Aoyama, T., Saito, S., Nam, H. and Kwon, O.: Comparison of Structured and Unstructured Mesh on Generic Helicopter Fuselage with a Rotor, Aerospace Numerical Simulation Symposium (ANSS), Tokyo, Japan, June 24-25, 2005.

16) Agarwal, R. K. and Dees, J. E.: Euler Caculations for Flowfield of a Helicopter Rotor in Hover, AIAA Paper 86-1782, 1986.

17) Kandil, O. A. and Chuang, A. H.: Computation of Steady and Unsteady Vortex Dominated Flows, AIAA Paper 87-1462, 1987.

18) Kandil, O. A. and Chuang, A. H.: Unsteady Transonic Airfoil Computation Using Implicit Euler Scheme on Body-Fixed Grid, AIAA J., 27, 8 (1989), pp. 1031-1037.

19) Sim, B. W. and Lim, J. W.: Bladee-Vortex Interaction (BVI) Noise \& Airload Prediction Using Loose Aerodynamic/Structural Coupling, American Helicopter Society 62nd Annual Forum, Phoenix, Arizona, 2006.

20) Peron, S., Benoit, C., Renaud, T., Sides, J., Tanabe, Y., Saito, S., Yang, C. and Aoyama, T.: ONERA/JAXA Common Investigations on CFD Tools for an Accurate Prediction of BVI, International Forum on Rotorcraft Multidisciplinary Technology, AHS Specialists' Conference, Seoul, Korea, October 15-17, 2007.

21) Aoyama, T., Kawachi, K. and Saito, S.: Unsteady Calculation for
Flowfield of Helicopter Rotor with Various Tip Shapes, 18th European Rotorcraft Forum, Paper No. B03, Avignon, France, September 1992.

22) Yamamoto, S. and Daiguji, H.: Higher-Order-Accurate Upwind Schemes for Solving the Compressible Euler and Navier-Stokes Equations, J. Comput. Fluids, 22 (1993), pp. 259-270.

23) Shima, E. and Jounouchi, T.: Role of CFD in Aeronautical Engineering (No. 14)_AUSM Type Upwind Schemes-, NAL SP-34, 1999, pp. $7-12$.

\section{Appendix}

\section{A. Translating coordinate transformation}

If we assume a 2-D translating reference frame without rotation with the Galilei transform assumption, $t=t^{\prime}$, as shown in Fig. A1, then the derivatives in space and time can be written using the chain rule as follows:

$$
\begin{aligned}
\frac{\partial}{\partial x} & =\frac{\partial}{\partial x^{\prime}} \frac{\partial x^{\prime}}{\partial x}+\frac{\partial}{\partial y^{\prime}} \frac{\partial y^{\prime}}{\partial x}+\frac{\partial}{\partial t^{\prime}} \frac{\partial t^{\prime}}{\partial x} \\
& =\frac{\partial}{\partial x^{\prime}} \frac{\partial\left(x-R_{x}\right)}{\partial x}+\frac{\partial}{\partial y^{\prime}} \frac{\partial\left(y-R_{y}\right)}{\partial x}+\frac{\partial}{\partial t^{\prime}} \frac{\partial t^{\prime}}{\partial x}=\frac{\partial}{\partial x^{\prime}} \\
\frac{\partial}{\partial y} & =\frac{\partial}{\partial x^{\prime}} \frac{\partial x^{\prime}}{\partial y}+\frac{\partial}{\partial y^{\prime}} \frac{\partial y^{\prime}}{\partial y}+\frac{\partial}{\partial t^{\prime}} \frac{\partial t^{\prime}}{\partial y} \\
& =\frac{\partial}{\partial x^{\prime}} \frac{\partial\left(x-R_{x}\right)}{\partial y}+\frac{\partial}{\partial y^{\prime}} \frac{\partial\left(y-R_{y}\right)}{\partial y}+\frac{\partial}{\partial t^{\prime}} \frac{\partial t^{\prime}}{\partial y}=\frac{\partial}{\partial y^{\prime}} \\
\frac{\partial}{\partial t} & =\frac{\partial}{\partial x^{\prime}} \frac{\partial x^{\prime}}{\partial t}+\frac{\partial}{\partial y^{\prime}} \frac{\partial y^{\prime}}{\partial t}+\frac{\partial}{\partial t^{\prime}} \frac{\partial t^{\prime}}{\partial t} \\
& =\frac{\partial}{\partial x^{\prime}} \frac{\partial\left(x-R_{x}\right)}{\partial t}+\frac{\partial}{\partial y^{\prime}} \frac{\partial\left(y-R_{y}\right)}{\partial t}+\frac{\partial}{\partial t^{\prime}} \frac{\partial t^{\prime}}{\partial t} \\
& =\frac{\partial}{\partial x^{\prime}} \frac{\partial x}{\partial t}-\frac{\partial}{\partial x^{\prime}} \frac{\partial R_{x}}{\partial t}+\frac{\partial}{\partial y^{\prime}} \frac{\partial y}{\partial t}-\frac{\partial}{\partial y^{\prime}} \frac{\partial R_{y}}{\partial t}+\frac{\partial}{\partial t^{\prime}} \frac{\partial t^{\prime}}{\partial t} \\
& =\frac{\partial}{\partial t^{\prime}}-u_{\mathrm{t}} \frac{\partial}{\partial x^{\prime}}-v_{\mathrm{t}} \frac{\partial}{\partial y^{\prime}}=\frac{\partial}{\partial t^{\prime}}-\vec{V}_{\mathrm{t}} \cdot \nabla \cdot
\end{aligned}
$$

In these terms,

$$
\frac{\partial R_{x}}{\partial x}=\frac{\partial R_{x}}{\partial y}=\frac{\partial R_{y}}{\partial x}=\frac{\partial R_{y}}{\partial y}=0
$$

and

$$
\frac{\partial t^{\prime}}{\partial x}=\frac{\partial t}{\partial x}=\frac{\partial t^{\prime}}{\partial y}=\frac{\partial t}{\partial y}=\frac{\partial y}{\partial x}=\frac{\partial x}{\partial y}=0
$$

because $\vec{R}=\vec{R}(t)$, and $x, y, t\left(=t^{\prime}\right)$ are independent variables.

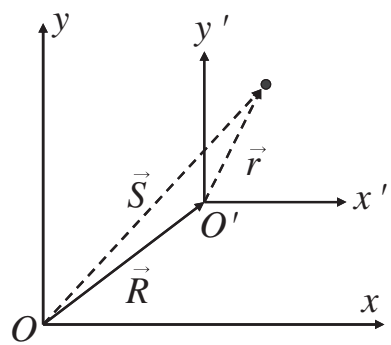

Fig. A1. 2-D translating reference frame without rotation. 


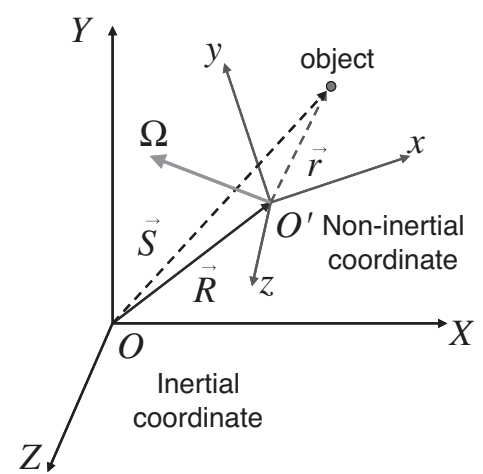

Fig. B1. Non-inertial (translating and rotating) coordinate.

\section{B. Coordinate transformation for non-inertial coor- dinate}

To derive the governing equations for a non-inertial (translating and rotating) coordinate as shown in Fig. B1, the geometry must be represented vectorially to give the absolute acceleration relative to some inertial coordinate system $X Y Z, \vec{S}=\vec{r}+\vec{R}$, where $\vec{S}$ is the position vector of a particle with respect to the fixed inertial frame $X Y Z, \vec{r}$ is the position vector of a particle with respect to the moving (translating \& rotating) non-inertial frame $x y z, \vec{R}$ is the posi- tion vector of the origin of moving non-inertial frame $x y z$, as shown in Fig. 3. A first differentiation of the absolute displacement of the particle gives the absolute velocity,

$$
\vec{V}=\vec{V}_{\mathrm{r}}+\frac{\mathrm{d} \vec{R}}{\mathrm{~d} t}+\vec{\varpi} \times \vec{r}=\vec{V}_{\mathrm{r}}+\vec{V}_{\mathrm{t}}+\vec{\varpi} \times \vec{r}=\vec{V}_{\mathrm{r}}+\vec{V}_{\mathrm{T}}
$$

where, $\vec{V}$ is the absolute velocity in inertial frame $X Y Z, \vec{V}_{\mathrm{r}}$ is the relative velocity in non-inertial frame $x y z, \vec{V}_{\mathrm{t}}$ is the translation velocity of the origin of $x y z, \vec{\varpi}$ is the angular velocity of rotating non-inertial frame $x y z$, and $\vec{V}_{\mathrm{T}}$ is the transformation velocity of $x y z$ from the absolute to the relative motion.

A second differentiation of the absolute displacement of the particle gives the absolute acceleration,

$$
\begin{aligned}
\vec{a} & =\frac{\mathrm{d} \vec{V}_{\mathrm{r}}}{\mathrm{d} t}+\frac{\mathrm{d}^{2} \vec{R}}{\mathrm{~d} t^{2}}+\frac{\mathrm{d} \vec{\varpi}}{\mathrm{d} t} \times \vec{r}+2 \vec{\varpi} \times \vec{V}_{\mathrm{r}}+\vec{\varpi} \times(\vec{\varpi} \times \vec{r}) \\
& =\vec{a}_{\mathrm{r}}+\vec{a}_{\mathrm{R}}
\end{aligned}
$$

where $\frac{\mathrm{d}^{2} \vec{R}}{\mathrm{~d} t^{2}}$ is the acceleration of the non-inertial origin of the coordinate $x y z, \frac{\mathrm{d} \vec{\omega}}{\mathrm{d} t} \times \vec{r}$ is the angular-acceleration effect, $2 \vec{\omega} \times \vec{V}_{\mathrm{r}}$ is the Coriolis acceleration, and $\vec{\varpi} \times(\vec{\varpi} \times$ $\vec{r})$ is the centrifugal acceleration. 